\title{
Isoprenaline as an aid to the induction of catecholamine dependent supraventricular tachycardias during programmed stimulation
}

\author{
BEATRICE BREMBILLA-PERROT, A TERRIER DE LA CHAISE, MARC PICHENÉ, \\ ETIENNE ALIOT, FRANÇOIS CHERRIER, CLAUDE PERNOT
}

From the Department of Cardiology, Brabois Hospital, Vandoeuvre lès Nancy, France

SUMMARY The effects of isoprenaline on the induction of supraventricular tachycardia by programmed stimulation were studied in 67 patients to see whether they correlated with $\underset{\infty}{\infty}$ spontaneous catecholamine mediated symptoms during exercise testing and Holter monitoring. Thirty seven control patients (group 1) did not have spontaneous arrhythmias either during exercise testing or Holter monitoring. Thirty patients (group 2) had documented exercise or stress $\rightarrow$ related supraventricular tachycardias-that is paroxysmal junctional tachycardia (24) or atrial arrhythmia (6). Programmed electrical stimulation was performed before and during the infusion $\vec{\varphi}$ of isoprenaline. No group 1 patient developed sustained supraventricular tachycardia during $0^{\infty}$ isoprenaline infusion. In 21 patients with paroxysmal junctional tachycardia and all the patients with atrial arrhythmias electrical stimulation during isoprenaline infusion produced the same tachycardia that had been seen during exercise testing and Holter monitoring. Changes in electrophysiological variables and the concentrations of serum potassium were not associated with the induction of supraventricular tachycardia by isoprenaline. Infusion of isoprenaline safely facilitated the induction of supraventricular tachycardia by programmed stimulation in patients who had spontaneously occurring catecholamine mediated symptoms.

Although isoprenaline is known to facilitate the induction of ventricular tachycardia ${ }^{2}$ it has rarely been used to aid the electrical induction of supraventricular tachycardia.

We report the results obtained when small infusions of isoprenaline were given to patients who did not have clinical supraventricular tachycardia and in whom standard stimulation techniques failed to induce tachycardia and to patients who did have spontaneous but non-inducible tachycardia.

\section{Patients and methods}

\section{PATIENTS}

Sixty seven patients who underwent programmed stimulation of the heart were classified according to the presence or absence of clinical and inducible supraventricular tachycardias.

Requests for reprints to Dr Béatrice Brembilla-Perrot, Department of Cardiologie, CHU de Brabois, 54500 Vandoeuvre lès Nancy, France.

Accepted for publication 1 November 1988

\section{Group 1}

Group 1 consisted of 37 patients aged from 12 to 67 (average 45) who had no clinical history of tachycar-? dia. Seventeen had underlying heart disease: dilated 3 cardiomyopathy (three), hypertrophic cardiomyopathy (five), coronary heart disease (two), mitral valve prolapse (two), congenital heart disease 0 (four), valve disease (one). Four patients in group 1 had Wolff-Parkinson-White syndrome. Electrophysiological studies were performed to investigate the cause of syncope (12) or dizziness (17). These o studies, which included ventricular stimulation, $N$ were also completed in eight patients with under-N lying heart disease (four cases) or were performed $\sigma$ to assess the severity of Wolff-Parkinson-White syndrome (four cases). Twenty four hour Holter $\frac{C}{\Phi}$ monitoring showed no evidence of supraventricular $\stackrel{\oplus}{\rightleftharpoons}$ arrhythmias (three patients had atrial extrasystoles). 7 No tachycardia or atrial extrasystoles were seeno

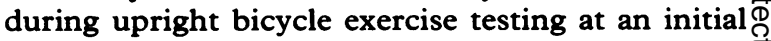
workload of $25 \mathrm{~W}$, with subsequent increases of $25 \mathrm{~W}$ every two minutes. Exercise continued until exhaustion or the onset of symptoms. 
No supraventricular tachycardia was induced by programmed stimulation in the basal state.

\section{Group 2}

Group 2 consisted of 30 patients aged from 15 to 80 (average 45) who had been admitted with supraventricular tachycardias and who had been included in a group of about 160 patients with supraventricular tachycardia studied in the laboratory over four years (1983 to 1987). Group 2a consisted of 24 patients with paroxysmal regular junctional reciprocating tachycardia and group $2 b$ consisted of six patients with paroxysmal atrial arrhythmias-atrial fibrillation (two), atrial flutter (one), and atrial tachycardia (three). All the patients in group 2 had tachycardia induced by exercise or psychological stress. In most an electrocardiographic recording of the tachycardia was available. None the less, only eight of the 29 patients who exercised on the bicycle showed supraventricular arrhythmias during exercise. There were frequent salvoes of atrial extrasystoles (five), atrial tachycardia (one), and paroxysmal junctional tachycardia (two) immediately after exercise. Twenty four hour Holter monitoring in all the patients showed a paroxysmal junctional tachycardia in nine patients and atrial tachycardia in three patients. Nine patients had underlying heart disease: mitral valve prolapse (six), valvar heart disease (one), and dilated cardiomyopathy (two). Eight patients had Wolff-Parkinson-White syndrome. Electrophysiological study was performed to investigate the mechanism of the tachycardia (23) or syncope associated with tachycardia (seven).

All patients had normal serum concentrations of potassium. Cardioactive drugs were stopped at least five half lives before the study.

\section{ELECTROPHYSIOLOGICAL STUDIES}

Electrophysiological studies were performed in nonsedated patients in the post-absorptive state after they had given their informed consent. All patients were in normal sinus rhythm at the time of study. Right heart catheterisation was performed through the femoral vein with three 6 or 7 French multielectrode catheters. Electrograms were recorded from the right atrium, the atrioventricular (AV) junction (His bundle electrogram), and the coronary sinus. Atrial and ventricular stimulation was performed with a programmable digital stimulator (Explorer 2000 Ela Médical) that delivered rectangular pulses lasting $1.8 \mathrm{~ms}$ at twice the diastolic threshold. Intracardiac electrograms and electrocardiographic leads I, III, and VI were simultaneously displayed on a multichannel oscilloscope (Siemens recording system) and recorded at a paper speed of 25 to $100 \mathrm{~mm} / \mathrm{s}$. Arterial blood pressure was measured by sphygmomanometry every five minutes.

We used the following protocol of programmed stimulation: $(a)$ incremental atrial pacing up to the onset of type 1 second degree block; $(b)$ atrial extrastimulus testing during sinus rhythm (S2) and atrial pacing at cycle lengths of 600 and $400 \mathrm{~ms}$ (S1 S2); (c) incremental ventricular pacing up to the onset of retrograde ventriculoatrial (VA) second degree or higher grades of ventriculoatrial block (maximal pacing rate 220 beats $/ \mathrm{min}$ ); (d) ventricular extrastimulus techniques up to $S 3$ during sinus rhythm and ventricular pacing (cycle lengths of 600 and $400 \mathrm{~ms}$ ) with two stimuli. In 21 patients the plasma concentration of potassium was measured after programmed stimulation in 21 patients.

Isoprenaline infusion was started at $0.5 \mu \mathrm{g} / \mathrm{min}$ and adjusted to increase the basal heart rate by at least $20 \%$. In all patients the heart rate was increased to at least 100 beats/min (maximum of 150 beats $/ \mathrm{min}$ ). During the infusion we repeated the stimulation protocol and measured the serum concentration of potassium at comparable cycle lengths. The effective refractory periods were determined at a cycle length of $400 \mathrm{~ms}$. The amount of isoprenaline required to induce the desired changes in heart rate ranged from 6 to $20 \mu \mathrm{g}$.

Heart rate and blood pressure were monitored continuously throughout the study.

\section{DEFINITION OF TERMS}

Non-sustained supraventricular tachycardia: at least five consecutive atrial extrasystoles, but less than one minute of tachycardia reproducibly induced.

Sustained supraventricular tachycardia: tachycardia that lasted more than one minute.

Non-sustained ventricular tachycardia: at least five consecutive ventricular extrasystoles, but reproducibly induced for less than one minute.

Sustained ventricular tachycardia: tachycardia that lasted more than one minute.

\section{STATISTICAL ANALYSIS}

We used a two tailed $t$ test for independent or paired samples. Numerical data are presented as mean (1 SD).

\section{Results}

Tables 1-3 show the results of electrophysiological testing in all the study groups.

ELECTROPHYSIOLOGICAL VARIABLES (TABLE 4) The effects of isoprenaline on heart rate and the AH and $\mathrm{HV}$ intervals were similar to those previously reported. ${ }^{3}$ Isoprenaline shortened the length of the sinus cycle in group 1 and in group 2. It also 
Table 1 Results of electrophysiological study before and after infusion of isoprenaline (Iso)

\begin{tabular}{|c|c|c|c|c|c|c|c|c|c|c|c|c|c|}
\hline Case & Sex & $\begin{array}{l}\text { Age } \\
(y r)\end{array}$ & Indic & $E C G$ & $H D$ & $E T$ & Holter & $C L(m s)$ & $A H(m s)$ & $\begin{array}{l}\text { Ant } \\
2 n d^{\circ} \text { block } \\
\text { (beats } / \text { min) }\end{array}$ & $\begin{array}{l}\text { Rctr } \\
\text { block } \\
\text { (beats/min) }\end{array}$ & A Stim & $\begin{array}{l}K+ \\
(m m c\end{array}$ \\
\hline 1 & $\mathbf{M}$ & 60 & $S$ & $\mathbf{N}$ & $\mathrm{HCM}$ & - & - & $\begin{array}{r}850 \\
\text { Iso } 500\end{array}$ & $\begin{array}{r}120 \\
60\end{array}$ & $\begin{array}{l}100 \\
160\end{array}$ & $\begin{array}{r}0 \\
200\end{array}$ & - & \\
\hline 2 & $\mathbf{M}$ & 48 & $\mathbf{S}$ & $\mathbf{N}$ & - & - & - & $\begin{array}{r}1000 \\
\text { Iso } 470\end{array}$ & $\begin{array}{l}70 \\
50\end{array}$ & $\begin{array}{l}160 \\
190\end{array}$ & $\begin{array}{r}0 \\
200\end{array}$ & = & \\
\hline 3 & $\mathbf{F}$ & 66 & $\mathbf{S}$ & $\mathbf{N}$ & - & - & - & $\begin{array}{l}650 \\
\text { Iso } \quad 500\end{array}$ & $\begin{array}{l}50 \\
50\end{array}$ & $\begin{array}{l}170 \\
230\end{array}$ & $\begin{array}{l}160 \\
200\end{array}$ & $\overline{\mathrm{AESS}}$ & \\
\hline 4 & $\mathbf{M}$ & 67 & $S$ & SB & - & - & - & $\begin{array}{r}1300 \\
\text { Iso } \quad 650\end{array}$ & $\begin{array}{l}75 \\
70\end{array}$ & 120 & $\begin{array}{r}90 \\
100\end{array}$ & $\overline{-}$ & \\
\hline 5 & $\mathbf{M}$ & 62 & $\mathbf{S}$ & MA & CHD & - & - & $\begin{array}{r}750 \\
\text { Iso } \quad 550\end{array}$ & $\begin{array}{l}70 \\
60\end{array}$ & $\begin{array}{l}205 \\
230\end{array}$ & $\begin{array}{l}0 \\
0\end{array}$ & 二 & \\
\hline 6 & $\mathbf{F}$ & 63 & D & $\mathbf{N}$ & Valv & - & - & $\begin{array}{r}620 \\
\text { Iso } 480\end{array}$ & $\begin{array}{l}70 \\
60\end{array}$ & $\begin{array}{l}220 \\
300\end{array}$ & $\begin{array}{r}0 \\
200\end{array}$ & $\bar{z}$ & \\
\hline 7 & $\mathbf{M}$ & 51 & D & SB & - & - & - & Iso $\begin{array}{r}1100 \\
600\end{array}$ & $\begin{array}{l}80 \\
60\end{array}$ & $\begin{array}{l}100 \\
210\end{array}$ & $\begin{array}{l}100 \\
230\end{array}$ & $\begin{array}{l}\mathrm{E} \\
\mathrm{E}\end{array}$ & $\begin{array}{l}3.9 \\
3.5\end{array}$ \\
\hline $\begin{array}{l}8 \\
0\end{array}$ & $\mathbf{M}$ & 66 & $S$ & LBBB & - & - & - & $\begin{array}{rr}780 \\
\text { Iso } \quad 650\end{array}$ & $\begin{array}{l}70 \\
70 \\
50\end{array}$ & $\begin{array}{l}160 \\
260\end{array}$ & $\begin{array}{r}160 \\
220\end{array}$ & $\overline{-}$ & $\begin{array}{l}4 \cdot 3 \\
4 \cdot 1\end{array}$ \\
\hline 9 & $\mathbf{M}$ & 30 & D & $\mathrm{N}$ & - & - & - & Iso $\begin{array}{r}650 \\
450\end{array}$ & $\begin{array}{l}50 \\
40\end{array}$ & $\begin{array}{l}230 \\
320\end{array}$ & $\begin{array}{r}0 \\
240\end{array}$ & $\overline{\mathrm{E}}$ & \\
\hline 10 & $\begin{array}{l}\mathrm{M} \\
\mathrm{F}\end{array}$ & 37 & D & $\mathbf{N}$ & - & VESs & VESs & Iso $\begin{array}{r}550 \\
400\end{array}$ & $\begin{array}{l}60 \\
30\end{array}$ & $\begin{array}{l}240 \\
290\end{array}$ & $\begin{array}{l}210 \\
220\end{array}$ & $\stackrel{\text { NSAF }}{-}$ & $\begin{array}{l}3 \cdot 5 \\
3 \cdot 4 \\
3.0\end{array}$ \\
\hline 11 & F & 62 & D & $\mathbf{N}$ & HCM & - & - & Iso $\begin{array}{r}1140 \\
600\end{array}$ & $\begin{array}{r}90 \\
80\end{array}$ & $\begin{array}{l}150 \\
230\end{array}$ & $\begin{array}{l}130 \\
220\end{array}$ & $\bar{z}$ & $\begin{array}{l}3.9 \\
3.9\end{array}$ \\
\hline 12 & $\mathbf{M}$ & 46 & $\mathbf{S}$ & $\mathbf{N}$ & - & - & - & $\begin{array}{r}920 \\
\text { Iso } \quad 750\end{array}$ & $\begin{array}{r}100 \\
70\end{array}$ & $\begin{array}{l}170 \\
250\end{array}$ & $\begin{array}{l}110 \\
180\end{array}$ & $\frac{E}{-}$ & $\begin{array}{l}3 \cdot 8 \\
3 \cdot 1\end{array}$ \\
\hline 13 & $\mathbf{M}$ & 49 & D & $N$ & HCM & VESs & VESs & $\begin{array}{r}1000 \\
\text { Iso } 440\end{array}$ & $\begin{array}{l}40 \\
65\end{array}$ & $\begin{array}{l}210 \\
260\end{array}$ & $\begin{array}{l}130 \\
220\end{array}$ & $\underline{E}$ & $\begin{array}{l}4 \cdot 3 \\
3 \cdot 6\end{array}$ \\
\hline 14 & $\mathbf{F}$ & 40 & $\mathbf{S}$ & $\mathbf{N}$ & - & - & VESs & $\begin{array}{r}800 \\
\text { Iso } 500\end{array}$ & $\begin{array}{r}75 \\
70 \\
100\end{array}$ & $\begin{array}{l}160 \\
240 \\
140\end{array}$ & $\begin{array}{r}0 \\
180\end{array}$ & $\overline{\overline{N S A}}$ & \\
\hline 15 & $M$ & 35 & S & $\mathbf{N}$ & - & VESs & VESs & $\begin{array}{r}800 \\
\text { Iso } \quad 400\end{array}$ & $\begin{array}{r}100 \\
70\end{array}$ & $\begin{array}{l}140 \\
210\end{array}$ & $\begin{array}{l}0 \\
0\end{array}$ & $\underline{\text { NSAF }}$ & \\
\hline 16 & $\mathbf{F}$ & 64 & D & $\mathrm{N}$ & - & - & VESs & $\begin{array}{r}1050 \\
\text { Iso } 700\end{array}$ & $\underset{50}{\mathrm{~J}}$ & $\begin{array}{l}170 \\
200\end{array}$ & $\begin{array}{l}140 \\
190\end{array}$ & $\bar{E}$ & \\
\hline 17 & $\mathbf{F}$ & $\begin{array}{l}36 \\
51\end{array}$ & D & $\begin{array}{l}\mathrm{N} \\
\mathrm{MI}\end{array}$ & RVD & - & $\begin{array}{l}\text { VESs } \\
-\end{array}$ & Iso $\begin{array}{r}450 \\
870\end{array}$ & $\begin{array}{l}50 \\
40 \\
80\end{array}$ & $\begin{array}{l}240 \\
300 \\
170\end{array}$ & $\begin{array}{r}0 \\
200\end{array}$ & $\underline{E}$ & \\
\hline 19 & F & 12 & syst & RVH & CHD & VESs & - & Iso $\begin{array}{r}550 \\
750\end{array}$ & $\begin{array}{r}80 \\
60 \\
110\end{array}$ & $\begin{array}{l}170 \\
250 \\
170\end{array}$ & $\begin{array}{r}170 \\
200 \\
0\end{array}$ & E & \\
\hline 19 & F & 12 & syst & KVH & cong & VEOS & & Iso 400 & 90 & 300 & 0 & - & \\
\hline 20 & $\mathbf{M}$ & 61 & syst & RBBB & $\mathrm{HCH}$ & - & VESs & 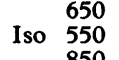 & $\begin{array}{r}75 \\
75\end{array}$ & $\begin{array}{l}190 \\
250\end{array}$ & $\begin{array}{l}110 \\
220\end{array}$ & - & \\
\hline 21 & M & 30 & D & $\mathbf{N}$ & DCM & - & - & $\begin{array}{r}850 \\
\text { Iso } 500\end{array}$ & $\begin{array}{r}100 \\
60\end{array}$ & $\begin{array}{l}150 \\
230\end{array}$ & $\begin{array}{l}0 \\
0\end{array}$ & - & $\begin{array}{l}4 \cdot 9 \\
3 \cdot 7\end{array}$ \\
\hline 22 & $\mathbf{M}$ & 56 & $S$ & $\mathbf{N}$ & - & - & VESs & $\begin{array}{r}660 \\
\text { Iso } \quad 500\end{array}$ & $\begin{array}{r}100 \\
60\end{array}$ & $\begin{array}{l}150 \\
230\end{array}$ & $\begin{array}{l}0 \\
0\end{array}$ & - & \\
\hline 23 & $\mathbf{M}$ & 42 & syst & LVH & DCM & - & VESs & $\begin{array}{r}600 \\
\text { Iso } \quad 540\end{array}$ & $\begin{array}{l}40 \\
80\end{array}$ & 200 & $\begin{array}{l}170 \\
200\end{array}$ & - & $\begin{array}{l}3 \cdot 6 \\
3 \cdot 3\end{array}$ \\
\hline 24 & $\mathbf{F}$ & 50 & D & $\mathrm{N}$ & - & - & VESs & $\begin{array}{r}700 \\
\text { Iso } \quad 450\end{array}$ & $\begin{array}{r}75 \\
60 \\
\end{array}$ & $\begin{array}{l}210 \\
230\end{array}$ & $\begin{array}{r}0 \\
220\end{array}$ & $\overline{-}$ & $\begin{array}{l}4 \cdot 1 \\
3 \cdot 7\end{array}$ \\
\hline 25 & F & 16 & D & $\mathbf{N}$ & RVD & VESs & VESs & 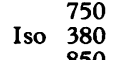 & $\begin{array}{r}100 \\
80\end{array}$ & $\begin{array}{l}150 \\
280\end{array}$ & $\begin{array}{r}0 \\
240\end{array}$ & $\begin{array}{l}\text { NSAF } \\
-\end{array}$ & $\begin{array}{l}3 \cdot 8 \\
3\end{array}$ \\
\hline 26 & $M$ & 56 & D & $\mathbf{N}$ & - & - & - & 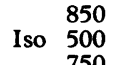 & $\begin{array}{r}120 \\
60\end{array}$ & $\begin{array}{l}100 \\
160\end{array}$ & $\begin{array}{r}0 \\
200\end{array}$ & $\bar{E}$ & \\
\hline 27 & $\mathbf{F}$ & 56 & D & $\mathrm{N}$ & - & - & - & $\begin{array}{r}750 \\
\text { Iso } \quad 500\end{array}$ & $\begin{array}{l}70 \\
60\end{array}$ & $\begin{array}{l}160 \\
200\end{array}$ & 190 & $\underline{\mathrm{E}}$ & \\
\hline 28 & $\mathbf{M}$ & 17 & $S$ & $\mathrm{~N}$ & MVP & - & VESs & $\begin{array}{r}950 \\
\text { Iso } \quad 500\end{array}$ & $\begin{array}{l}90 \\
70\end{array}$ & $\begin{array}{l}150 \\
210\end{array}$ & $\begin{array}{l}100 \\
150\end{array}$ & E & \\
\hline 29 & $\mathbf{F}$ & 59 & D & $\mathbf{N}$ & RVD & VESs & VESs & $\begin{array}{r}750 \\
\text { Iso } 400\end{array}$ & $\begin{array}{l}90 \\
70\end{array}$ & $\begin{array}{l}190 \\
220\end{array}$ & $\begin{array}{l}0 \\
0\end{array}$ & - & \\
\hline 30 & $\mathbf{M}$ & 46 & $\mathbf{S}$ & LVH & HCM & - & - & $\begin{array}{r}950 \\
\text { Iso } 510\end{array}$ & $\begin{array}{l}95 \\
65\end{array}$ & $\begin{array}{l}170 \\
220\end{array}$ & $\begin{array}{r}0 \\
120\end{array}$ & - & \\
\hline 31 & $\mathbf{M}$ & 51 & syst & LVH & DCM & - & - & 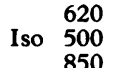 & $\begin{array}{l}80 \\
65 \\
75\end{array}$ & $\begin{array}{l}180 \\
210\end{array}$ & $\begin{array}{l}190 \\
220\end{array}$ & $\overline{\bar{Z}}$ & $\begin{array}{l}3.7 \\
2.9\end{array}$ \\
\hline 32 & $\mathbf{M}$ & 37 & D & $\mathrm{N}$ & MVP & - & VESs & Iso $\begin{array}{r}850 \\
500\end{array}$ & $\begin{array}{l}75 \\
60\end{array}$ & $\begin{array}{l}180 \\
260\end{array}$ & $\begin{array}{l}130 \\
190\end{array}$ & NSAF & $\begin{array}{l}4.6 \\
3.8\end{array}$ \\
\hline 33 & $\mathbf{M}$ & 2 & $\mathbf{S}$ & $\mathbf{N}$ & HCM & - & - & $\begin{array}{r}1050 \\
\text { Iso } 550\end{array}$ & $\begin{array}{l}70 \\
55\end{array}$ & $\begin{array}{l}110 \\
250\end{array}$ & $\begin{array}{r}0 \\
190\end{array}$ & $\underline{\text { NSAF }}$ & $\begin{array}{l}6 \cdot 1 \\
4 \cdot 6\end{array}$ \\
\hline 34 & $\mathbf{M}$ & 37 & syst & WPW & - & - & VESs & $\begin{array}{r}750 \\
\text { Iso } \quad 400\end{array}$ & $\begin{array}{l}70 \\
60\end{array}$ & $\begin{array}{l}190 \\
280\end{array}$ & $\begin{array}{l}190 \\
280\end{array}$ & $\bar{z}$ & \\
\hline 35 & $\mathbf{F}$ & 25 & syst & WPW & - & - & - & $\begin{array}{r}750 \\
\text { Iso } \quad 350\end{array}$ & $\begin{array}{l}80 \\
50\end{array}$ & $\begin{array}{l}180 \\
300\end{array}$ & $\begin{array}{l}180 \\
300\end{array}$ & $\begin{array}{l}\mathbf{E} \\
\mathbf{E}\end{array}$ & \\
\hline 36 & $\mathbf{M}$ & 35 & syst & WPW & - & - & - & Iso $\begin{array}{r}800 \\
600\end{array}$ & $\begin{array}{l}50 \\
50\end{array}$ & $\begin{array}{l}250 \\
280\end{array}$ & $\begin{array}{l}250 \\
280\end{array}$ & 二 & \\
\hline 37 & $\mathbf{M}$ & 14 & syst & WPW & - & - & - & $\begin{array}{l}520 \\
\text { Iso } \quad 400\end{array}$ & $\begin{array}{l}75 \\
50\end{array}$ & $\begin{array}{l}220 \\
280\end{array}$ & $\begin{array}{l}220 \\
280\end{array}$ & NSAF & \\
\hline
\end{tabular}

Indic (indication)-S, syncope; $\mathrm{D}$, dizziness; syst, systematically (to study tachycardia induction).

ECG (electrocardiogram)-N, normal; SB, sinus bradycardia; MI, myocardial infarction; LBBB, left bundle branch block; LVH, left ventric hypertrophy; RBBB, right bundle branch block; RVH, right ventricular hypertrophy.

HD (heart disease)-HCM, hypertrophic cardiomyopathy; CHD, coronary heart disease; RVD, right ventricular dysplasia; cong, congenital $\mathrm{F}$. DCM, dilated cardiomyopathy; MVP, mitral valve prolapse.

ET (exercise testing)-VES, ventricular extrasystole.

CL, cycle length; Ant 2 nd ${ }^{\circ}$ block, heart rate at which atrioventricular second degree block occurred; Retr block, heart rate at which ventriculoatrial block occurred; A Stim, results of programmed atrial stimulation; AES, atrial extrasystole; E, repetitive atrial echo beat; NSAF, non-sustained atrial fibrillation. 
Table 2 Patients with exercise related paroxysmal junctional tachycardias (group 2a)

\begin{tabular}{|c|c|c|c|c|c|c|c|c|c|c|c|c|c|}
\hline Case & Sex & $\begin{array}{l}\text { Age } \\
(y r)\end{array}$ & Indic & $E C G$ & $H D$ & $E T$ & Holter & $C L(m s)$ & $A H(m s)$ & $\begin{array}{l}\text { Ant } \\
\text { 2nd block } \\
\text { (beats } / \text { min) }\end{array}$ & $\begin{array}{l}\text { Retr } \\
\text { block } \\
\text { (beats } / \text { min) }\end{array}$ & & \\
\hline 1 & $\mathrm{~F}$ & 24 & $\mathrm{~T}$ & WPW & MVP & - & - & $\begin{array}{r}100 \\
\text { Iso } 500\end{array}$ & $\begin{array}{r}100 \\
80\end{array}$ & $\begin{array}{l}120 \\
240\end{array}$ & $\begin{array}{l}200 \\
200\end{array}$ & $\underset{\text { SPIT }}{\mathrm{E}}$ & \\
\hline 2 & $\mathrm{~F}$ & 59 & $\mathrm{~T}$ & WPW & - & - & - & 900 & 90 & 140 & 190 & $\bar{c}$ & \\
\hline 3 & $M$ & 28 & $\mathrm{~T}$ & $\mathrm{~N}$ & - & - & - & Iso 450 & $\begin{array}{l}60 \\
90\end{array}$ & $\begin{array}{l}250 \\
160\end{array}$ & $\begin{array}{r}200 \\
0\end{array}$ & ${ }_{-}^{\text {SPJT }}$ & \\
\hline 4 & $M$ & 55 & $\mathrm{~T}$ & $\mathrm{~N}$ & - & - & - & $\begin{array}{r}1 \text { so } 450 \\
900\end{array}$ & $\begin{array}{l}60 \\
70\end{array}$ & $\begin{array}{l}260 \\
185\end{array}$ & $\begin{array}{l}200 \\
150\end{array}$ & $=$ & \\
\hline 5 & $M$ & 31 & $\mathrm{~T}-\mathrm{S}$ & $\mathrm{N}$ & - & - & - & $\begin{array}{r}\text { Iso } 500 \\
870 \\
\text { Iso } 450\end{array}$ & $\begin{array}{l}60 \\
90 \\
70\end{array}$ & $\begin{array}{l}210 \\
180 \\
250\end{array}$ & $\begin{array}{l}200 \\
140 \\
200\end{array}$ & $\begin{array}{l}\text { SPJT } \\
\text { NSAT } \\
\text { SPIT }\end{array}$ & \\
\hline 6 & $M$ & 57 & $\mathrm{~T}$ & WPW & MVP & - & PJT & $\begin{array}{l}1000 \\
\text { Iso } 450\end{array}$ & $\begin{array}{l}70 \\
60\end{array}$ & $\begin{array}{l}150 \\
250\end{array}$ & $\begin{array}{l}200 \\
200\end{array}$ & $\begin{array}{l}\text { NSPJT } \\
\text { SPJT }\end{array}$ & \\
\hline 7 & $\mathrm{~F}$ & 33 & $\mathrm{~T}-\mathrm{S}$ & WPW & - & - & - & $\begin{array}{r}750 \\
\text { Iso } 350\end{array}$ & $\begin{array}{l}60 \\
45\end{array}$ & $\begin{array}{l}150 \\
300\end{array}$ & $\begin{array}{l}180 \\
200\end{array}$ & $\begin{array}{l}\mathrm{E} \\
\text { NSPIT }\end{array}$ & \\
\hline 8 & $\mathrm{~F}$ & 33 & $\mathrm{~T}$ & WPW & - & - & - & $\begin{array}{l}1000 \\
\text { Iso } 500\end{array}$ & $\begin{array}{r}130 \\
70\end{array}$ & $\begin{array}{l}120 \\
230\end{array}$ & $\begin{array}{l}160 \\
200\end{array}$ & $\begin{array}{l}\text { NSPJT } \\
\text { SPJT }\end{array}$ & \\
\hline 9 & $\mathrm{~F}$ & 60 & $\mathrm{~T}-\mathrm{S}$ & WPW & - & - & - & $\begin{array}{r}700 \\
\text { Iso } 450\end{array}$ & $\begin{array}{l}80 \\
50\end{array}$ & $\begin{array}{l}180 \\
240\end{array}$ & $\begin{array}{l}160 \\
200\end{array}$ & $\begin{array}{l}\text { NSAFI } \\
\text { SPJT }\end{array}$ & \\
\hline 10 & $M$ & 34 & $\mathrm{~T}$ & RBBB & MVP & - & PJT & $\begin{array}{r}960 \\
\text { Iso } 500\end{array}$ & $\begin{array}{l}90 \\
70\end{array}$ & $\begin{array}{l}150 \\
250\end{array}$ & $\begin{array}{r}0 \\
170\end{array}$ & SPJT & \\
\hline 11 & $\mathrm{~F}$ & 80 & $\mathrm{~T}-\mathrm{S}$ & $\mathrm{N}$ & - & $?$ & PJT & $\begin{array}{r}600 \\
\text { Iso } 500\end{array}$ & $\begin{array}{l}90 \\
80\end{array}$ & $\begin{array}{l}170 \\
190\end{array}$ & $\begin{array}{r}0 \\
160\end{array}$ & $\begin{array}{l}\text { NSAT } \\
\text { SPJT }\end{array}$ & \\
\hline 12 & $M$ & 15 & $\mathrm{~T}$ & $\mathrm{~N}$ & - & - & - & $\begin{array}{r}860 \\
\text { Iso } 500\end{array}$ & $\begin{array}{l}70 \\
50\end{array}$ & $\begin{array}{l}145 \\
210\end{array}$ & $\begin{array}{l}170 \\
200\end{array}$ & $\overline{\text { SPJT }}$ & \\
\hline 13 & $\mathrm{~F}$ & 61 & $\mathrm{~T}$ & $\mathrm{~N}$ & MVP & - & PJT & $\begin{array}{c}1000 \\
\text { Iso } 700\end{array}$ & $\begin{array}{r}130 \\
70\end{array}$ & $\begin{array}{l}135 \\
230\end{array}$ & $\begin{array}{l}170 \\
200\end{array}$ & $\begin{array}{l}\text { NSPJT } \\
\text { SPJT }\end{array}$ & \\
\hline 14 & $M$ & 33 & $\mathrm{~T}$ & $\mathrm{~N}$ & - & - & - & $\begin{array}{r}770 \\
\text { Iso } 550\end{array}$ & $\begin{array}{l}75 \\
60\end{array}$ & $\begin{array}{l}215 \\
240\end{array}$ & $\begin{array}{l}180 \\
240\end{array}$ & $\begin{array}{l}\text { NSPJT } \\
\text { SPJT }\end{array}$ & \\
\hline 15 & $M$ & 29 & $\mathrm{~T}$ & WPW & - & $\begin{array}{l}\text { PJT } \\
\text { (post ET) }\end{array}$ & PJT & $\begin{array}{r}800 \\
\text { Iso } 420\end{array}$ & $\begin{array}{l}60 \\
40\end{array}$ & $\begin{array}{l}160 \\
230\end{array}$ & $\begin{array}{l}210 \\
210\end{array}$ & 二 & \\
\hline 16 & $\mathrm{~F}$ & 31 & $\mathrm{~T}$ & $\mathrm{~N}$ & - & - & - & $\begin{array}{r}660 \\
\text { Iso } 500\end{array}$ & $\begin{array}{r}110 \\
60\end{array}$ & $\begin{array}{l}170 \\
270\end{array}$ & $\begin{array}{l}120 \\
220\end{array}$ & $\stackrel{\text { O }}{\text { SPJT }}$ & \\
\hline 17 & $M$ & 26 & $\mathrm{~T}$ & $\mathrm{~N}$ & - & - & - & $\begin{array}{r}800 \\
\text { Iso } 420\end{array}$ & $\begin{array}{l}80 \\
50\end{array}$ & $\begin{array}{l}150 \\
250\end{array}$ & $\begin{array}{l}180 \\
240\end{array}$ & $\begin{array}{l}\mathrm{E} \\
\text { SPJT }\end{array}$ & $\begin{array}{l}4 \cdot 2 \\
2 \cdot 7\end{array}$ \\
\hline 18 & $M$ & 46 & $\mathrm{~T}$ & LVH & DCM & AESs & PJT & $\begin{array}{r}640 \\
\text { Iso } 420\end{array}$ & $\begin{array}{l}90 \\
70\end{array}$ & $\begin{array}{l}220 \\
240\end{array}$ & 200 & SPJT & $\begin{array}{l}4 \\
3.5\end{array}$ \\
\hline 19 & $M$ & 52 & $\mathrm{~T}$ & LVH & DCM & - & VESs & $\begin{array}{l}800 \\
550\end{array}$ & $\begin{array}{l}70 \\
60\end{array}$ & $\begin{array}{l}150 \\
210\end{array}$ & $\begin{array}{l}150 \\
220\end{array}$ & $\begin{array}{l}\text { NSA f } \\
\text { SPJT }\end{array}$ & $\begin{array}{l}4.5 \\
3.5\end{array}$ \\
\hline 20 & $M$ & 64 & $\mathrm{~T}-\mathrm{S}$ & $\mathrm{N}$ & - & AESs & $\mathrm{AT}$ & $\begin{array}{r}750 \\
\text { Iso } 450\end{array}$ & $\begin{array}{l}60 \\
45\end{array}$ & $\begin{array}{l}230 \\
310\end{array}$ & $\begin{array}{l}180 \\
230\end{array}$ & $\begin{array}{l}\text { E } \\
\text { SPJT }\end{array}$ & $\begin{array}{l}3.4 \\
3.5\end{array}$ \\
\hline 21 & $\mathrm{~F}$ & 45 & $\mathrm{~T}$ & $\mathrm{~N}$ & - & - & $\begin{array}{l}\text { PJT } \\
\text { VT }\end{array}$ & $\begin{array}{r}710 \\
\text { Iso } 400\end{array}$ & $\begin{array}{l}75 \\
35\end{array}$ & $\begin{array}{l}220 \\
280\end{array}$ & $\begin{array}{l}150 \\
240\end{array}$ & $\begin{array}{l}\text { NSAF } \\
\text { SPJT }\end{array}$ & $\begin{array}{l}3.5 \\
3.3\end{array}$ \\
\hline 22 & $\mathrm{~F}$ & 33 & $\mathrm{~T}-\mathrm{D}$ & WPW & - & $\begin{array}{l}\text { PJT } \\
\text { (post ET) }\end{array}$ & PJ'T & $\begin{array}{r}800 \\
\text { Iso } 430\end{array}$ & $\begin{array}{l}45 \\
40\end{array}$ & $\begin{array}{l}210 \\
290\end{array}$ & $\begin{array}{l}130 \\
220\end{array}$ & $\begin{array}{l}\text { NSAF } \\
\text { SPJT }\end{array}$ & $\begin{array}{l}3.5 \\
3.3\end{array}$ \\
\hline 23 & $\mathrm{~F}$ & 61 & $\mathrm{~T}$ & $\mathrm{~N}$ & MVP & - & $\begin{array}{l}\text { NS PJ'T } \\
\text { VESs }\end{array}$ & $\begin{array}{l}1000 \\
\text { Iso } 570\end{array}$ & $\begin{array}{r}140 \\
90\end{array}$ & $\begin{array}{l}140 \\
210\end{array}$ & $\begin{array}{r}0 \\
160\end{array}$ & $\begin{array}{l}\text { NSAFI } \\
\text { SPJT }\end{array}$ & $\begin{array}{l}4 \cdot 1 \\
2 \cdot 7\end{array}$ \\
\hline 24 & $\mathrm{~F}$ & 43 & $\mathrm{~T}$ & $\mathrm{~N}$ & - & - & - & $\begin{array}{r}720 \\
\text { Iso } 500\end{array}$ & $\begin{array}{l}60 \\
60\end{array}$ & $\begin{array}{l}200 \\
300\end{array}$ & $\begin{array}{r}170 \\
>240\end{array}$ & $\begin{array}{l}\text { NSPJT } \\
\text { SPJT }\end{array}$ & $\begin{array}{l}3.4 \\
3.4\end{array}$ \\
\hline
\end{tabular}

WPW. Wolff-Parkinson-White; AT, atrial tachycardia; PJT, paroxysmal junctional tachycardia; S, sustained: NS, non-sustained: AF, atrial fibrillation: gfl. atrial flutter. See footnote to table I for other abbreviations.

Table 3 Results of electrophysiological study before and after infusion of isoprenaline in patients with exercise related paroxysmal atrial arrhythmias (group $2 b$ )

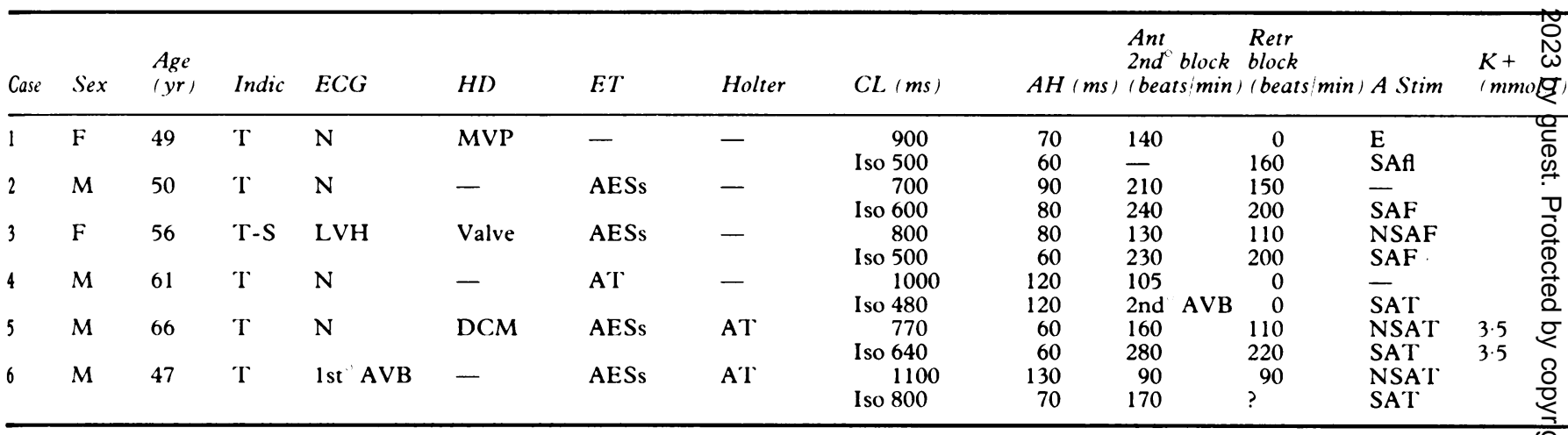


Table 4 Electrophysiological variables (mean (1SD) before and after isoprenaline (Iso)

\begin{tabular}{|c|c|c|c|c|c|}
\hline & $C L(m s)$ & $A H$ (ms) & $\begin{array}{l}\text { Ant } 2 n d^{\circ} \text { block } \\
\text { (beats/min) }\end{array}$ & $\begin{array}{l}\text { Retr block } \\
\text { (beats/min) }\end{array}$ & $\begin{array}{l}\mathrm{K}^{+} \\
(\mathrm{mmol} / \mathrm{l})\end{array}$ \\
\hline $\begin{array}{l}\text { Group 1: } \\
\text { Before } \\
\text { Iso } \\
\text { p }\end{array}$ & $\begin{array}{l}812(75) \\
505(91) \\
<0 \cdot 001\end{array}$ & $\begin{array}{l}82(21) \\
62(13) \\
<0.001\end{array}$ & $\begin{array}{l}173(39) \\
243(40) \\
<0.001\end{array}$ & $\begin{array}{l}104(51) \\
177(64) \\
<0.001\end{array}$ & $\begin{array}{l}4.2(0.7) \\
3.6(0.7) \\
<0.03\end{array}$ \\
\hline $\begin{array}{l}\text { Group 2: } \\
\text { Before } \\
\text { Iso } \\
\text { p }\end{array}$ & $\begin{array}{l}832(123) \\
485(70) \\
<0.001\end{array}$ & $\begin{array}{l}86(23) \\
63(18) \\
<0.001\end{array}$ & $\begin{array}{l}169(32) \\
245(31) \\
<0.001\end{array}$ & $\begin{array}{l}143(52) \\
204(37) \\
<0.001\end{array}$ & $\begin{array}{c}3.9(0.4) \\
3.3(0.4) \\
<0.01\end{array}$ \\
\hline
\end{tabular}

shortened the $\mathrm{AH}$ interval in group 1 and in group 2 and had no effect on the HV interval. Isoprenaline reduced the atrial effective refractory period calculated at a driven atrial rhythm of $400 \mathrm{~ms}$ in both group $1(218(12) \mathrm{ms} v 171(14) \mathrm{ms}, \mathrm{p}<0.01)$ and group $2(209(16) \mathrm{ms} v 165(19) \mathrm{ms}, \mathrm{p}<0.01)$. The atrial rate at which anterograde type 1 second degree block developed increased significantly in group 1 and group 2 . The ventricular rate at which retrograde second degree block developed increased significantly in group 1 and group 2. Isoprenaline reduced the ventricular effective refractory periods calculated at a driven rhythm of $400 \mathrm{~ms}$ from 210 (5) $\mathrm{ms}$ to 175 (7) $\mathrm{ms}(\mathrm{p}<0.01)$ in group 2 and from $202(7) \mathrm{ms}$ to $173(5) \mathrm{ms}(\mathrm{p}<0.01)$ in group 2. The effects of isoprenaline on the anterograde and retrograde refractory periods of Kent bundles were similar to those reported elsewhere ${ }^{4}$ : isoprenaline generally shortened the refractory periods in patients with the longest initial values (average $50 \mathrm{~ms}$ ).

SERUM CONCENTRATIONS OF POTASSIUM

The serum concentration of potassium fell significantly in group 1 .

\section{INDUCED SUPRAVENTRICULAR TACHYCARDIA}

In the basal state sustained supraventricular tachycardia could not be induced by programmed stimulation in any patient; a non-sustained atrial arrhythmia was induced in five patients from group 1 and in eight from group 2 and a non-sustained paroxysmal junctional tachycardia was induced in five patients from group 2 .

After infusion of isoprenaline sustained supraventricular tachycardia could not be initiated in any group 1 patients, even when they had had nonsustained supraventricular tachycardia during the basal study. Non-sustained atrial tachycardia was induced in one group 1 patient who did not have inducible arrhythmia in the basal state.

After isoprenaline sustained junctional tachycardia was induced in 21 patients in group 2a either spontaneously (three) or by programmed stimulation
(18). There were 13 re-entrant tachycardias in the atrioventricular node and eight re-entrant tachycar- $\rightarrow$ dias conducted retrogradely through a Kent bundle. $+\overrightarrow{+}$ Non-sustained junctional tachycardia was induced in $\underset{\infty}{\stackrel{\omega}{\perp}}$ one patient.

After isoprenaline, clinical sustained atrial 윽 tachycardia could be reproduced in six patients in $\vec{D}$ group 2 either spontaneously (three) or by programmed stimulation (three). There was one atrial flutter, two atrial fibrillations, and three atrial tachycardias.

In group 2 the sensitivity of the isoprenaline test, defined as the percentage of inducible sustained supraventricular tachycardia, was $90 \%$. Specificity, defined as the percentage of negative tests, was $100 \%$ in group 1.

\section{CORRELATION BETWEEN JUNCTIONAL}

TACHYCARDIA AND ELECTROPHYSIOLOGICAL

VARIABLES AND SERUM POTASSIUM (TABLE 5)

We analysed certain variables in patients in group $2 \mathrm{a}$ and group 1 to discover whether any of them correlated with the induction of junctional tachycardia.

After isoprenaline we did not find any differences between groups 1 and $2 \mathrm{a}$ for variation in cycle length, AH interval, heart rate at which anterograde type 1 second degree block developed, and serum potas- 0 sium. In group 2 a retrograde block was significantly more common after electrophysiological testing in the basal state $(p<0.05)$ and after isoprenaline $(p<$ 0.02 ) (fig). However, the occurrence of a fast $\mathrm{N}$ retrograde conduction after isoprenaline did not $\mathrm{N}$ assist the prediction of the induction of junctional tachycardia: seven patients from group 1 without $\frac{\omega}{\sigma}$ retrograde conduction in the basal state showed retrograde conduction on isoprenaline at stimulation rates of up to $200 / \mathrm{min}$. The sensitivity of the isoprenaline test defined as the percentage of patients $\square$ in whom retrograde conduction developed at stimulation rates of $200 / \mathrm{min}$ was $86 \%$ in group $2 a, \overrightarrow{\mathbb{D}}$ but its specificity, defined as the percentage that $\vec{D}$ developed retrograde conduction at rates of $<200 \frac{0}{\sigma}$ beats $/ \mathrm{min}$ was $38 \%$ in group 1 . 
Table 5 Electrophysiological variables (mean (1SD) before and after isoprenaline in groups 1 and $2 a$

\begin{tabular}{|c|c|c|c|}
\hline & Group 1 & Group $2 a$ & $p$ \\
\hline \multicolumn{4}{|l|}{ CL (ms): } \\
\hline Before & $812(75)$ & $832(131)$ & \\
\hline $\begin{array}{c}\text { Iso } \\
\mathrm{AH} \text { (ms): }\end{array}$ & $505(91)$ & $483(73)$ & NS \\
\hline \multicolumn{4}{|l|}{$\mathrm{AH}(\mathrm{ms})$ : } \\
\hline Iso & $62(13)$ & $63(18)$ & NS \\
\hline \multicolumn{4}{|l|}{ Ant 2nd ${ }^{\circ}$ block } \\
\hline Before & $173(39)$ & $169(35)$ & NS \\
\hline & $243(40)$ & $245(33)$ & NS \\
\hline \multicolumn{4}{|l|}{ Retr block } \\
\hline Before & $104(51)$ & $144(65)$ & $<0.005$ \\
\hline \multicolumn{4}{|l|}{$\mathrm{K}^{+}$Iso $(\mathrm{mmol} / \mathrm{l})$} \\
\hline $\begin{array}{l}\text { Before } \\
\text { Iso }\end{array}$ & $\begin{array}{l}4.2(0.7) \\
3.6(0.5)\end{array}$ & $\begin{array}{l}3.9(0.4) \\
3.3(0.4)\end{array}$ & $\begin{array}{l}\text { NS } \\
\text { NS }\end{array}$ \\
\hline
\end{tabular}

$\mathrm{CL}$, cycle length; Ant $2 \mathrm{nd}^{\circ}$ block, heart rate at which atrioventricular second degree block occurred; Retr block, heart rate at which ventriculoatrial block occurred; $\mathrm{K}^{+}$, serum concentration of potassium.

ADVERSE EFFECTS OF THE ISOPRENALINE TEST Arterial blood pressure fell significantly in only one patient. Three patients in group 1 and one in group 2 showed transient sinus bradycardia and a fall in blood pressure several minutes after the beginning of

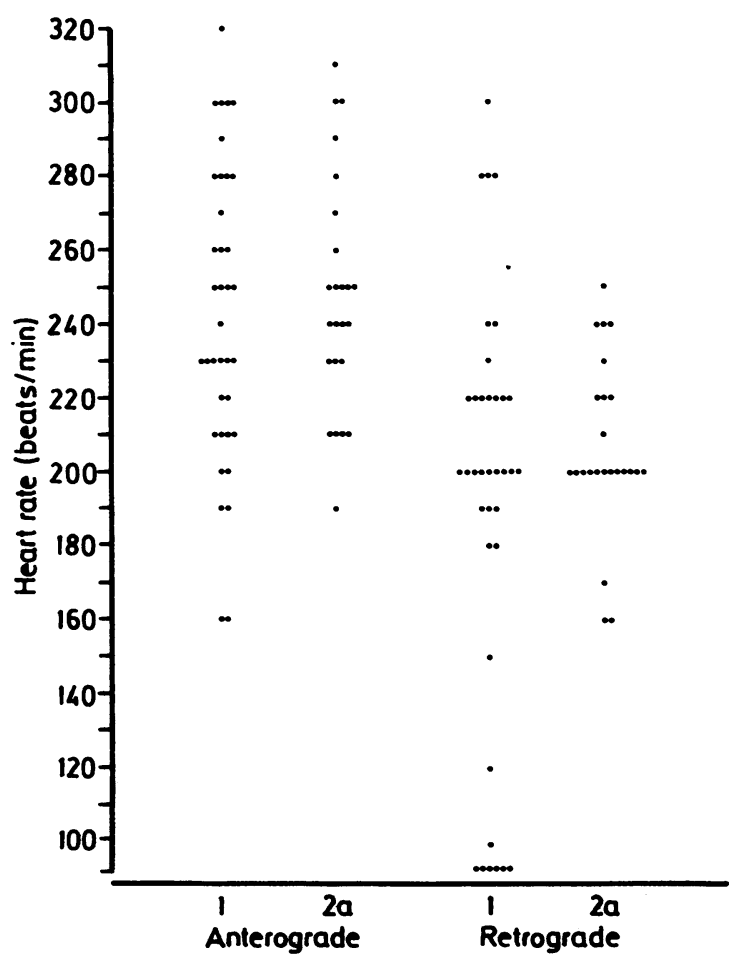

Figure Heart rates at which anterograde and retrograde type 1 second degree block developed during isoprenaline infusion in groups 1 and $2 a$. isoprenaline infusion; one of these patients had spontaneous salvoes of atrial tachycardia.

One patient from group 2 had spontaneous nonsustained uniform ventricular tachycardia (130 beats $/ \mathrm{min}$ ). The systematic programmed ventricular stimulation induced non-sustained multiform ventricular tachycardia in the basal state in two patients in group 1 and four in group 2. After isoprenaline, none of the patients from group 1 and only two from group 2 had induced multiform non-sustained ventricular tachycardia.

\section{Discussion}

We found that the isoprenaline test assisted the diagnosis of catecholamine mediated junctional tachycardias (sensitivity $90 \%$, specificity $100 \%$ ).

The role of the sympathetic nervous system in supraventricular arrhythmias was suggested by clinical observations, the bicycle exercise test, and 24 hour Holter monitoring. ${ }^{5}$ Adrenergic supraventricular tachycardias are induced by exercise or stress, and they occur predominantly during the day. There have been few studies of adrenergic supraventricular tachycardia. The role of adrenergic effects, which modify the refractory periods of accessory pathways, has been studied mainly in Wolff-Parkinson-White syndrome. ${ }^{467}$ Coumel et al reported Holter studies to evaluate the role of the sympathetic nervous system in arrhythmias other than in the Wolff-Parkinson-White syndrome. ${ }^{5}$ In our study, the sensitivity of Holter monitoring for the diagnosis of adrenergic supraventricular arrhythmia was poor $(40 \%)$. The sensitivity of exercise testing for triggering a supraventricular tachycardia was also poor $(40 \%)$. Moreover, when tachycardia occurs immediately after exercise during Holter monitoring, as in cases 15 and 22, the mechanism could be adrenergic or vagal. ${ }^{8}$

These findings indicated the desirability of a pharmacological test that induced trachycardias caused by adrenergic mechanisms. In patients with exercise induced ventricular arrhythmias isoprenaline often caused identical arrhythmia. ${ }^{910}$ Isoprenaline produces $\beta$ adrenergic stimulation, which affects the electrophysiological properties of the single cell and the intact heart in several ways. ${ }^{1-11}$ In single fibres isoprenaline increased the spontaneous phase IV depolarisation of sinus node, facilitated the development of triggered automaticity, and shortened the action potential duration. In intact hearts, isoprenaline increased the sinus rate and the rate of ectopic pacemakers, accelerated atrioventricular conduction, and shortened the refractoriness of the atrioventricular node and His-Purkinje system. It might be difficult to establish whether the tachycar- 
dia induced by isoprenaline resulted from direct stimulation of cardiac $\beta$ adrenoreceptors or from reflex changes, which might be in the opposite direction, depending on whether the blood pressure rose or fell. ${ }^{12}$ Vagal tone may be increased, as in three of our patients, or reduced by the haemodynamic effects of isoprenaline.

An alternative approach to the adrenergic initiation of tachycardia is to use atropine to withdraw the vagal effect, leaving the action of natural catecholamine unopposed. A pharmacological test could also be based on adrenaline, noradrenaline, and atropine.

The frequency with which isoprenaline facilitates the induction of atrial tachycardia has not been reported before. In our study there were six patients with an adrenergic mediated tachycardia that could be reproduced by infusion of isoprenaline and electrophysiological stimulation. The spontaneous occurrence of the arrhythmia in three of them may implicate enhanced automaticity. Non-sustained atrial tachycardia was induced in only one of the controls. Thus infusion of isoprenaline seems a useful provocative test to detect adrenergic paroxysmal atrial tachycardia.

There are few studies of the frequency with which isoprenaline facilitates the induction of junctional tachycardia. Levy et al and Hariman et al reported two cases of catecholamine dependent atrioventricular nodal reentrant tachycardia ${ }^{1314}$; Hariman et al proposed the enhancement of ventriculoatrial conduction as the mechanism of these tachycardias. ${ }^{14}$ In our study, this mechanism was not confirmed. Most reports deal with the induction of junctional tachycardia associated with the Wolff-ParkinsonWhite syndrome. The spontaneous initiation of reciprocating tachycardia in the Wolff-ParkinsonWhite syndrome by isoprenaline infusion was reported by Krikler et al. ${ }^{15}$ Kuck et al found a strong correlation between the results of isoprenaline infusion at rest and the results of an exercise test, ${ }^{16}$ and in five patients with exercise induced junctional tachycardia, isoprenaline infusion identified the arrhythmia. On the other hand, Brugada et al did not find any correlation between the occurrence of isoprenaline induced tachycardias and exercise induced tachycardia. ${ }^{17}$ The same results were reported for supraventricular tachycardias. ${ }^{12}$ The effects of isoprenaline and exercise on the facilitation of arrhythmia do not always coincide: isoprenaline facilitated the induction of arrhythmia in patients with sustained ventricular tachycardia not related to exertion. ${ }^{2}$

In patients who did not have Wolff-ParkinsonWhite syndrome or spontaneous arrhythmias, we did not find that infusion of isoprenaline increased the induction of non-sustained atrial or ventricular tachycardia. The mechanism by which isoprenaline facilitates the induction of junctional tachycardia remains unknown. Triggered automaticity and delayed after-depolarisation were possible mechanisms for the tachycardia that occurred during isoprenaline infusion in the two patients with spontaneous junctional tachycardia. In the study of Krikler et al incessant attacks of tachycardia without antecedent PR prolongation were induced by isoprenaline and were attributed to the speeding of the sinus rate, which might sufficiently shorten the refractory periods of the atrial and anomalous pathways to permit retrograde conduction up the anomalous pathway to the atrium thus causing an echo beat and tachycardia. ${ }^{15}$ In most of our patients $(n=19)$, the arrhythmias required an initiation beat and were stopped by one or two stimuli, suggesting that isoprenaline infusion facilitated reentry by reducing the refractoriness and increasing conduction velocity and the myocardium. The induction of catecholamine dependent reentrant tachycardia has been attributed to a rapid anterograde and/or retrograde conduction ${ }^{14}{ }^{18}$; this explanation was not confirmed in our study.

Because adrenaline can reproduce stress hypokalaemia ${ }^{1920}$ another mechanism by which isopren aline might have facilitated reentry was by a reduction in intracellular potassium. The fall in serum potassium, however, was similar in groups 1 and 2 , so this mechanism cannot account for the facilitation of arrhythmia.

Infusion of isoprenaline can safely be used to facilitate the induction of supraventricular tachycardia in patients who have spontaneous supraventricular tachycardia, when sympathetic stimulation is enhanced.

We thank Dr R Lazzara for criticisms and advice.

\section{References}

1 Reddy CP, Gettes LS. Use of isoproterenol as an aid to electric induction of chronic recurrent ventricula tachycardia. Am J Cardiol 1979;77:705-13.

2 Freedman RA, Swerdlow CD, Echt DS, Winkle RA, Soderholm-Difatte V, Mason JW. Facilitation of ventricular tachyarrhythmia induction by isoproterenol. Am J Cardiol 1984;54:765-70.

3 Vargas G, Akhtar M, Damato AN. Electrophysiologic effects of isoproterenol on cardiac conduction system in man. Am Heart $J$ 1975;90:25-34.

4 Wellens HJJ, Brugada P, Roy D, Weiss J, Barr FW Effect of isoproterenol on the anterograde refractory 
period of the accessory pathway in patients with the Wolff-Parkinson-White syndrome. Am J Cardiol 1982;50:180-4.

5 Coumel Ph, Attuel P, Leclercq JF, Friocourt P. Arythmies ventriculaires d'origine vagale ou catécholergique. Effets comparés du traitement béta-bloquant et phénomène d'échappement. Arch Mal Coeur 1982; 75:373-88.

6 Levy S, Broustet JP, Clementy J, Vircoulon B, Guern $P$, Bricaud H. Syndrome de Wolff-Parkinson-White. Corrélations entre l'exploration électrophysiologique et l'effet de l'épreuve d'effort sur l'aspect électrocardiographique de préécitation. Arch Mal Coeur 1979; 72:634-40.

7 Strasberg B, Ashley WW, Wyndham CRC, et al. Treadmill exercise testing in the Wolff-ParkinsonWhite syndrome. Am J Cardiol 1980;45:742-8.

8 Dimsdale JE, Hartley H, Guiney T, Ruskin JN, Greenblatt D. Post exercise peril. JAMA 1984;251:630-2.

9 Gettes LS, Surawicz B. Long term prevention of paroxysmal arrhythmias with propranolol therapy. Am J Med Sci 1967;254:257-65.

10 Brembilla-Perrot B, Terrier de la Chaise A, Pichene M, Pernot $\mathrm{C}$, Cherrier $\mathrm{F}$. Valeur diagnostique du test à l'isuprel dans les tachycardies d'effort. Arch Mal Coeur 1986;79:302-13.

11 Kassebaum DG, Van Dyke AR. Electrophysiological effects of isoproterenol on Purkinje fibers of the heart. Circ Res 1966;19:940-6.

12 Arnold JMO, McDevitt DG. Contribution of the vagus to the haemodynamic responses following intravenous boluses of isoprenaline. Br J Clin Pharmacol 1983;15:423-9.
13 Levy S, Pierron JP, Eisinger JF, et al. Tachycardie supraventriculaire induite par l'effort et les cathé cholamines. Arch Mal Coeur 1985;78:1431-6.

14 Hariman RJ, Gomez JAC, El-Sherif N. Catecholamines-dependent atrioventricular nodal reentrant tachycardia. Circulation 1983;67:681-6.

15 Krikler D, Curry P, Attuel P, Coumel Ph. "Incessant" tachycardias in Wolff-Parkinson-White syndrome. 1: Initiation without antecedent extrasystoles or PR lengthening, with reference to reciprocation after shortening of cycle length. Br Heart J 1976;38: 885-96.

16 Kuck K-H, Kunze KP, Roewer N, Bleifeld W. Electrophysiologic studies after isoproterenol and at exercise in patients with exercise induced circus movement tachycardia [Abstract]. Circulation 1983; 68(suppl III):76.

17 Brugada $P$, Facchini $M$, Wellens $H J J$. Effects of isoproterenol and amiodarone and the role of exercise in initiation of circus movement tachycardia in the accessory atrioventricular pathway. Am J Cardiol 1986;57:146-9.

18 Waxman MB, Wald RW. Effects of autonomic tone on tachycardias. In: Surawicz B, Reddy CP, Prystowski EN, eds. Tachycardias. Boston: Martinus Nijhoff, 1984:67-99.

19 Brown MJ. Hypokalemia from beta 2-receptors stimulation by circulating epinephrine. $\mathrm{Am} J$ Cardiol 1985;56:3D-5D.

20 Struthers AD, Reid JL, Whitesmith R, Rodger JC. Effect of intravenous adrenaline on electrocardiogram, blood pressure, and serum potassium. Br Heart J 1983;49:90-3. 